\title{
Uso de Prefijos y Sufijos en Terminologia Anatomica. Un Análisis desde el Origen Etimológico
}

\author{
Use of Prefixes and Suffixes in Terminologia Anatomica. \\ An Analysis from an Etymological Origin
}

Nicolás Vidal-Seguel'; Marcela Pumeyrau-Solar²; Rodrigo Lizama-Pérez ${ }^{3,4}$ \& Bélgica Vásquez

\begin{abstract}
VIDAL-SEGUEL, N.; PUMEYRAU-SOLAR. M.; LIZAMA-PÉREZ, R. \& VÁSQUEZ, B. Uso de prefijos y sufijos en Terminologia Anatomica. Un análisis desde el origen etimológico. Int. J. Morphol., 39(3):698-704, 2021.

RESUMEN: El aprendizaje de la anatomía requiere que los estudiantes incorporen una gran cantidad de nuevo vocabulario, esto puede conllevar algunas dificultades debido al desconocimiento de la mayoría de estos términos. A pesar de los esfuerzos realizados para que el uso de la Terminologia Anatomica (TA) sea claro y preciso, la enseñanza de esta sigue siendo compleja. Bajo este contexto, se hace necesario innovar en nuevas estrategias que favorezcan el aprendizaje por parte de los estudiantes. Es por ello, que el objetivo de este estudio fue analizar, desde el origen etimológico, los prefijos y sufijos incluidos en $T A$, con el fin de facilitar los procesos de enseñanza y aprendizaje de la anatomía humana. El uso de cada prefijo y sufijo en anatomía fue explorado en la segunda edición de TA. Los afijos encontrados fueron analizados desde un punto de vista etimológico según las directrices propuestas por el Diccionario Médico-Biológico, Histórico y Etimológico de la Universidad de Salamanca. Como resultados se pudo corroborar un amplio uso de prefijos y sufijos en TA, además de constatar que todos los términos analizados, desde un punto de vista etimológico, concuerdan con su utilización actual en anatomía. Por lo tanto, el conocimiento y la comprensión de éstos, podría facilitar los procesos de enseñanza y aprendizaje de la anatomía humana.
\end{abstract}

PALABRAS CLAVE: Prefijos; Sufijos; Terminologia Anatomica; Educación; Anatomía Humana.

\section{INTRODUCCIÓN}

El aprendizaje de la anatomía requiere que los estudiantes incorporen una gran cantidad de nuevo vocabulario, esto puede conllevar algunas dificultades debido al desconocimiento de la mayoría de estos términos (Guiraldes et al., 2001). Estudios recientes demuestran la dificultad de los estudiantes para comprender y retener los términos anatómicos (Kulkarni, 2014; Singh et al., 2015), del mismo modo, un estudio realizado por Bergman et al., (2013) donde se evaluó la percepción de los estudiantes de medicina sobre un plan de estudio, reveló que muchos estudiantes catalogaban la anatomía como "aburrida" y creían que solo requería autodisciplina, más que una capacidad intelectual para aprender la gran cantidad de información. Esto, sumado, a que la principal estrategia de aprendizaje está basada en técnicas memorísticas, sin entender realmente el significado de los términos anatómicos (Stephens \& Moxham, 2016), resulta ser un problema complejo en el proceso de enseñanza aprendizaje de esta materia.
Ante esta problemática, es necesario innovar en estrategias que faciliten el aprendizaje de los términos anatómicos desde el estudio de Terminologia Anatomica (TA), la que se encuentra redactada en latín y es usada como base por la comunidad científica internacional (Sakai, 2007) con el fin de unificar todos los términos anatómicos empleados (Aziz et al., 2002). En los últimos años, algunas iniciativas han estado orientadas a utilizar el conocimiento etimológico y gramatical para facilitar la comprensión y el aprendizaje de $T A$ en el estudio de la anatomía humana (Moya et al., 2017; Vidal-Seguel et al., 2019, 2021).

Lo anterior, pone en manifiesto que la comprensión de cualquier término se facilita al descubrir los aspectos que lo configuran. Así, conocer la etimología de los términos anatómicos resulta necesario para mejorar la comprensión de la anatomía y su estudio. Este postulado se apoya en el reporte de Smith et al., (2007), quienes concluyeron que el conoci-

\footnotetext{
${ }^{1}$ Escuela de Enfermería, Facultad de Salud, Universidad Santo Tomás, Chile.

${ }^{2}$ Programa de Magíster en Lingüística Aplicada, Facultad de Humanidades y Arte, Universidad de Concepción, Concepción, Chile.

${ }^{3}$ Departamento de Ciencias Morfológicas, Facultad de Medicina y Ciencia, Universidad San Sebastián, General Lagos 1163, Valdivia 509000, Chile.

${ }^{4}$ Departamento de Educación Física y Deporte, Facultad de Ciencias del Deporte, Universidad de Granada, Granada, España.

${ }^{5}$ Facultad de Ciencias de la Salud, Universidad de Tarapacá, Iquique, Chile.
} 
miento básico de las etimologías latina y griega, hace más sencillo el aprendizaje y la utilización del lenguaje médico por parte de los estudiantes de las ciencias de la salud.

Desde una perspectiva gramatical, una palabra se compone de unidades mínimas significativas denominadas morfemas. El morfema central o núcleo de la palabra recibe el nombre de raíz o lexema, y posee un significado léxico o referencial. Del mismo modo, existen morfemas que se agregan a la raíz modificando su significado, llamados afijos, de los cuales existe un número limitado para cada lengua. Dentro de esta categoría es posible encontrar afijos que aparecen antes de la raíz, los que reciben el nombre de prefijos, y otros que se encuentran después de la raíz llamados sufijos. Existen tres clases de sufijos: 1) apreciativos, que son aquellos que entregan una cualidad a la palabra; 2) flexivos, los que tienen un rol netamente gramatical y 3) derivativos, que permiten cambiar la categoría gramatical de la raíz a la que se unen (Kroeger, 2005).

En anatomía, una gran cantidad de términos están conformados por al menos un afijo, teniendo estos un origen etimológico latín o griego (Jiménez, 2012). Es por ello, que conocer el significado de estos podría facilitar la comprensión y aprendizaje de una gran cantidad de términos anatómicos. Bajo este postulado, el objetivo de este estudio fue analizar, desde el origen etimológico, los prefijos y sufijos incluidos en $T A$, con el fin de facilitar los procesos de enseñanza y aprendizaje de la anatomía humana.

\section{MATERIAL Y MÉTODO}

El uso de cada prefijo y sufijo en anatomía fue explorado en la segunda edición de TA (FIPAT, 2019). Los afijos encontrados fueron analizados desde un punto de vista etimológico según las directrices propuestas por el Diccionario Médico-Biológico, Histórico y Etimológico de la Universidad de Salamanca (Cortés \& Ureña, 2019).

Finalmente, todos los datos recolectados fueron expuesto en tablas para representar de mejor manera los hallazgos encontrados y facilitar su análisis.

\section{RESULTADOS}

La presencia de prefijos y sufijos en $T A$ es alta y su utilización ayuda a dar sentido a distintos términos anatómicos, extendiéndose en la totalidad de los sistemas y regiones del cuerpo humano.
Los prefijos y sufijos utilizados en anatomía poseen un origen principalmente latino, sin embargo, un número no despreciable encuentran su origen en la lengua griega. Las Tablas I y II detallan el número de términos relacionados a cada prefijo y como ellos pueden tributar a muchos otros nombres anatómicos asociados.

El prefijo de origen latín más frecuente en la construcción de términos anatómicos corresponde a inter (entre) con 58 de ellos en TA (Tabla I) como, por ejemplo: intervertebral, intercostal e intercondílea. Desde cada uno de estos 58 términos emergen muchos más. Es así como desde el término "intercondílea" derivan: fosa intercondílea, línea intercondílea, área intercondílea anterior, área intercondílea posterior y eminencia intercondílea. Todas ellas sugerentes a una misma ubicación "entre cóndilos".

El prefijo de origen griego más frecuente en la construcción de términos anatómicos corresponde a para (junto a) con 40 de ellos en TA (Tabla II) como, por ejemplo: paraesternal, parafaríngea y parauretral. Al igual que lo mencionado anteriormente, desde cada término pueden emerger muchos más. Es así como desde el termino paraesternal derivan: pliegue paraesternal y ganglios paraesternales. Todos ellos sugerente a una ubicación "junto al esternón".

El análisis de TA demostró la presencia de las tres clases de sufijos anteriormente descritas. La Tabla III muestra solo los sufijos de tipo apreciativo, los que se subdividen en sufijos de tipo -diminutivos-, de -abundancia- y que -hace algo- (Cortés \& Ureña). Estos, al igual que los prefijos, son capaces de unirse a la raíz de una palabra entregándole un nuevo sentido.

Por otra parte, los sufijos de tipo flexivos cumplen una función principalmente gramatical, es decir, otorga una información respecto al género, número, persona verbal, entre otros. Un ejemplo de esto es el sufijo ae que indica plural y permite la construcción del término en latín glandulae (glándulas). Los sufijos de tipo derivativo ampliamente utilizado en TA cumplen la función de cambiar la categoría gramatical de la palabra, por ejemplo: pasar de un verbo a un sustantivo, como es el caso del sufijo on del término tendón [tend- lat. 'poner tenso' (verbo) + on(em) (sufijo) lat.]. Estas últimas dos clases de sufijos, flexivos y derivativos, no se analizaron en gran profundidad ya que, como se mencionó, su función es netamente gramatical, por lo que no ejerce un rol significativo en la asociación de los términos a través del análisis del origen etimológico de la palabra. 
Tabla I. Uso de prefijos de origen latino en Terminologia Anatomica.

\begin{tabular}{|c|c|c|c|}
\hline Prefijo & $\begin{array}{l}\text { Significado } \\
\text { etimológico }\end{array}$ & Frecuencia & Uso en anatomía \\
\hline bi & $\begin{array}{l}\text { bi(s) lat., 'dos', 'dos } \\
\text { veces' }\end{array}$ & 1 & Término anatómico con dos porciones: bíceps \\
\hline dis & $\begin{array}{l}d \bar{\imath}-/ d i s \text { lat., 'en distintas } \\
\text { direcciones' }\end{array}$ & 3 & $\begin{array}{l}\text { Términos referidos a una separación de una estructura anatómica: } \\
\text { distobucal, distopalatina y distolingual. }\end{array}$ \\
\hline extra & extrā lat., 'fuera de' & 5 & $\begin{array}{l}\text { Términos localizados fuera de una estructura anatómica: } \\
\text { extracapsulares, extraserosa, extraperitoneal, extraocular, etc. }\end{array}$ \\
\hline infra & infra lat., 'Debajo' & 18 & $\begin{array}{l}\text { Términos localizados debajo de una estructura anatómica: } \\
\text { infraorbitaria, infrapatelar, infraglótica, infrahioideos, etc. }\end{array}$ \\
\hline inter & inter lat., 'entre' & 58 & $\begin{array}{l}\text { Términos localizados entre dos estructuras anatómicas: } \\
\text { intervertebral, intercostal, interlobar, intermuscular, etc. }\end{array}$ \\
\hline intra & intrā lat., 'en el interior' & 21 & $\begin{array}{l}\text { Términos localizados en el interior de una estructura anatómica: } \\
\text { intracapsulares, intraauricular, intramural, intraparietal, etc. }\end{array}$ \\
\hline multi & multi lat., 'numeroso' & 3 & $\begin{array}{l}\text { Términos anatómicos con más de dos porciones: multipeniforme, } \\
\text { multífidos y multiforme. }\end{array}$ \\
\hline pre & prae lat., 'antes de & 32 & $\begin{array}{l}\text { Términos localizados delante de una estructura anatómica: } \\
\text { prevertebral, prefrontal, pretraqual, preaórtica. etc. }\end{array}$ \\
\hline semi & semi lat., 'Medio' 'mitad' & 8 & $\begin{array}{l}\text { Términos atribuidos a la mitad de una estructura anatómica: } \\
\text { semilunar, semiespinoso, semitenfinoso, etc. }\end{array}$ \\
\hline sub & sub/sus lat., 'debajo' & 40 & $\begin{array}{l}\text { Términos localizados bajo una estructura anatómica: subtálamo, } \\
\text { subaracnoideo, subclavio, subcostales, etc. }\end{array}$ \\
\hline super & super lat., 'encima' & 1 & $\begin{array}{l}\text { Término localizado encima de una estructura anatómica: } \\
\text { superciliar. }\end{array}$ \\
\hline supra & suprā lat., 'sobre' & 39 & $\begin{array}{l}\text { Términos localizados sobre una estructura anatómica: } \\
\text { supraorbitario, suprahioideos, suprarrenal, supraquiasmático, etc. }\end{array}$ \\
\hline
\end{tabular}

\section{DISCUSIÓN}

El objetivo del presente estudio fue analizar desde un punto de vista etimológico los prefijos y sufijos incluidos en TA. Se pudo corroborar el gran uso de estos afijos en la construcción de términos utilizados e incluidos en $T A$ y su presencia se extiende sobre todos los sistemas y regiones del cuerpo humano. Por lo tanto, el conocimiento y la comprensión de éstos, facilitará los procesos de enseñanza y aprendizaje de una gran cantidad de términos anatómicos.

La anatomía humana es reconocida como una asignatura compleja y muchas veces desmotivadora, debido a su abundante vocabulario (Torres, 2013). Sin embargo, el comprender los términos desde sus orígenes, contribuye a que estos tomen sentido y mayor comprensión (Moore et al., 2010). Si reflexionamos sobre la conformación del término nos resultará mucho más sencillo trasladar su significado de una manera eficaz y comprensible.
Dentro del amplio uso de prefijos se puede constatar que todos ellos son concordantes con su utilización en anatomía, es decir, cada prefijo se relaciona perfectamente con el uso que se le da en la descripción anatómica. Sin embargo, en la realización de este estudio se pueden poner bajo la lupa ciertos prefijos que aparentemente significan lo mismo, pero que podrían tener ciertas diferencias. Tales casos son: 1) sub - infra - hipo; 2) Supra - super - epi; 3) intra - endo.

Los prefijos sub, infra e hipo son definidos en las Tablas I y II como "debajo", "abajo" o "debajo de". Sin embargo, existen diferencias entre ellos. Los prefijos sub e infra son de origen latino e hipo de origen griego, a su vez los prefijos infra e hipo también pueden referirse a inferioridad "inferior de" o "bajo nivel de" y finalmente el prefijo hipo, además, pude hacer referencia a "escases de" (Real Academia Nacional de Medicina de España, 2011). 
VIDAL-SEGUEL, N.; PUMEYRAU-SOLAR. M.; LIZAMA-PÉREZ, R. \& VÁSQUEZ, B. Uso de prefijos y sufijos en Terminologia Anatomica. Un análisis desde el origen etimológico. Int. J. Morphol., 39(3):698-704, 2021.

Tabla II. Uso de prefijos de origen griego en Terminologia Anatomica.

\begin{tabular}{|c|c|c|c|c|}
\hline Prefijo & \multicolumn{2}{|c|}{$\begin{array}{l}\text { Significado } \\
\text { etimológico }\end{array}$} & Frecuencia & Uso en anatomía \\
\hline anti & $\begin{array}{l}\text { antí } \quad \text { óvií } \\
\text { 'frente a', 'con }\end{array}$ & $\begin{array}{l}\text { gr., } \\
\text { tra' }\end{array}$ & 3 & $\begin{array}{l}\text { Términos para nombra estructuras anatómicas localizadas } \\
\text { frente a otras: antihélix, antitrago y antitragohelicina. }\end{array}$ \\
\hline endo & $\begin{array}{l}\text { endo } \varepsilon \text { évoo } \\
\text { 'dentro' }\end{array}$ & gr., & 13 & $\begin{array}{l}\text { Términos para nombrar estructuras anatómicas localizadas } \\
\text { dentro de otras: endometrio, endoneuro, endomisio, etc. }\end{array}$ \\
\hline epi & $\begin{array}{l}\text { ep(i) Émí } \\
\text { 'sobre' }\end{array}$ & gr., & 14 & $\begin{array}{l}\text { Términos para nombrar estructuras anatómicas localizadas } \\
\text { sobre otras: epigástrica, epimisio, epineuro, etc. }\end{array}$ \\
\hline hemi & $\begin{array}{l}\text { hēmi } \quad \dot{\eta} \mu ı \\
\text { 'mitad', 'semi' }\end{array}$ & gr., & 2 & $\begin{array}{l}\text { Términos para nombrar estructuras anatómicas que representa } \\
\text { la mitad de un todo: hemiácigos y hemisferio. }\end{array}$ \\
\hline hipo & $\begin{array}{l}\text { hypó ímó } \\
\text { 'debajo de' }\end{array}$ & gr., & 6 & $\begin{array}{l}\text { Términos para nombrar estructuras anatómicas localizadas por } \\
\text { debajo de otras: hipotálamo, hipogloso, hipófisis, etc. }\end{array}$ \\
\hline meta & $\begin{array}{l}\text { metá } \mu \varepsilon т \alpha ́ \text { ' } \\
\text { 'después } \\
\text { 'posterior }\end{array}$ & $\begin{array}{l}\text { gr., } \\
\text { de', }\end{array}$ & 5 & $\begin{array}{l}\text { Términos para nombrar estructuras anatómicas localizadas más } \\
\text { allá de otras: metacarpo, metatarso, metáfisis, etc. }\end{array}$ \\
\hline para & $\begin{array}{l}\text { para mapó } \\
\text { 'junto a' } \\
\text { margen de' }\end{array}$ & gr. & 40 & $\begin{array}{l}\text { Términos para nombrar estructuras anatómicas localizadas } \\
\text { junto a o al margen de otras: paraesternal, parafaríngea, } \\
\text { parauretral, etc. }\end{array}$ \\
\hline pro & $\begin{array}{l}\text { pró } \quad \text { Tpó } \\
\text { 'delante } \\
\text { 'antes de' }\end{array}$ & $\begin{array}{l}\text { gr., } \\
\text { de', }\end{array}$ & 4 & $\begin{array}{l}\text { Términos referidos a proyecciones hacia delante de una } \\
\text { estructura anatómica: prominencia, prosencéfalo, promontorio. }\end{array}$ \\
\hline
\end{tabular}

Los prefijos supra, super y epi son definidos en las tablas I y II como "encima” o "sobre”. Sin embargo, existen ciertas diferencias entre ellos. Lo prefijos super y supra son de origen latín a diferencia del prefijo epi que es de origen griego; a su vez, el prefijo super no solo hace referencia a encima o sobre, sino que también, puede significar: 1) "preeminencia" o "excelencia", 2) "en grado sumo" o 3 ) "exceso", esto podría explicar su poco uso en TA (Real Academia Nacional de Medicina de España).

Los prefijos intra y endo son definidos en las Tablas I y II como "en el interior", "hacia el interior" y "dentro". La diferencia subyace en que uno es de origen latino (intra) y otro de origen griego (endo), sin embargo, su uso no parece tener mayores diferencias (Real Academia Nacional de Medicina de España).

Los sufijos de tipo apreciativos analizados en nuestro estudio, se diferencian de los otros tipos de sufijos, en su capacidad de unirse a la raíz sin que esto conlleve a una alteración del significado léxico de esta (Marcos, 1980). En nuestros resultados es posible encontrar los términos arteria y arteriola, donde el sufijo ola confiere la característica de pequeña, sin altera el significado principal que hace referencia a arteria. El sufijo ola forma parte de una serie de terminaciones utilizadas para dar característica de pequeño, los denominado sufijos -diminutivos- (Tabla III). Un resultado interesante es el sufijo ote que generalmente se utiliza para dar característica de grande, sin embargo, en TA es utilizado para la creación del término islote, islotes pancreáticos e islotes olfatorios, sugerente a una estructura de menor tamaño respeto a otra. Del mismo modo, la terminación illo (illu( $m$ ) latín) utilizada para la creación de términos como frenillo, conductillo, huesecillo y lobulillo, confiere característica de pequeño y es ampliamente usada en el castellano. Sin embargo, de los términos mencionados solo frenillo posee el sufijo en latín illum (Frenulum), por lo cual el uso de esta terminación, illo, puede ser sujeta a mayor análisis (Lizama-Pérez et al., 2020) y a futuras investigaciones.

Dentro de los sufijos de tipo apreciativo, nuestros resultados muestran los de -abundancia-, con el sufijo os, el que permite la construcción de los términos fibroso, membranoso, entre otros. Estos permiten otorgar el sentido de abundancia de fibras y membranas, respetivamente, dando origen a términos tales como: trígono fibroso derecho, trígono fibroso izquierdo, anillo fibroso derecho/izquierdo, laberinto membranoso, bursa del músculo semimembranoso. Todos ellos sugerente a una estructura abundante en fibras o membranas. 
Tabla III. Sufijos apreciativos en Terminologia Anatomica.

\begin{tabular}{|c|c|c|c|}
\hline Sufijos & Significado etimológico & $\begin{array}{c}\text { Principales usos en } \\
\text { anatomía }\end{array}$ & Ejemplos \\
\hline \multicolumn{4}{|c|}{ Diminutivos } \\
\hline ela, illa & $\begin{array}{l}\text { ella }(m) \text { lat., hace adj. o sust., } \\
\text { 'pequeño'. }\end{array}$ & rodilla, patela, glabela. & $\begin{array}{l}\text { rodilla: [rot(am) lat. 'rueda' + -ella }(m) \text { lat. } \\
\text { 'pequeño'] }\end{array}$ \\
\hline culo & cul(um) lat., 'pequeño'. & $\begin{array}{l}\text { tubérculo, } \quad \text { canalículo, } \\
\text { folículo, divertículo, } \\
\text { testículo, pedículo, colículo, } \\
\text { opérculo. }\end{array}$ & $\begin{array}{l}\text { tubérculo: [tūber lat. 'bulto', 'protuberancia' } \\
+- \text {-cul(um) lat. 'pequeño'] }\end{array}$ \\
\hline cula & cula $(m)$ lat., 'pequeña'. & clavícula, aurícula. & $\begin{array}{l}\text { clavícula: [clāu(em) lat. 'llave' + -cula }(m) \\
\text { lat. 'pequeña'] }\end{array}$ \\
\hline illo, illa & $\begin{array}{l}\text { illu(m) lat., hace adj. o sust., } \\
\text { 'pequeño'. }\end{array}$ & $\begin{array}{l}\text { frenillo, lobulillo, } \\
\text { conductillo, huesecillos. }\end{array}$ & $\begin{array}{l}\text { frenillo: [frēnu }(m) \text { lat. 'freno', 'brida' + - } \\
\text { illu }(m) \text { lat. 'pequeño'] }\end{array}$ \\
\hline elo & $\begin{array}{l}\text { ell-u(m)/-a(m) lat., hace adj. } \\
\text { o sust., 'pequeño' }\end{array}$ & cerebelo & $\begin{array}{l}\text { cerebelo: [cereb(rum) lat. 'cerebro' + -ell- } \\
u(m) /-a(m) \text { lat. 'pequeño'] }\end{array}$ \\
\hline ola & $\begin{array}{l}\text { ol-a }(m) \text { lat., hace adj. o } \\
\text { sust., 'pequeño' }\end{array}$ & arteriola, areola. & $\begin{array}{l}\text { arteriola: [artēri(ā) d́ónpía gr. 'tubo', } \\
\text { 'arteria' + -ol-a(m) lat. 'pequeño'] }\end{array}$ \\
\hline olo & $\begin{array}{l}\text { ol-u }(m) \text { lat., hace adj. o } \\
\text { sust., 'pequeño'. }\end{array}$ & $\begin{array}{l}\text { alveolo, } \\
\text { bronquiolo. }\end{array}$ & $\begin{array}{l}\text { alveolo: [alue(um) lat. 'recipiente' + -ol- } u(m) \\
\text { lat. 'pequeño'] }\end{array}$ \\
\hline ote, ota & $\begin{array}{l}\text { ote lat., puede aportar una } \\
\text { variación muy amplia de } \\
\text { valores: aumentativos y } \\
\text { diminutivos }\end{array}$ & islote & isla pequeña \\
\hline ula & $\begin{array}{l}\text { ula }(m) \text { lat., hace adj. o sust., } \\
\text { 'pequeño'. }\end{array}$ & $\begin{array}{l}\text { cápsula, glándula, vesícula, } \\
\text { vénula. }\end{array}$ & [caps(am) lat. 'caja' + -ula $(m)$ lat. 'pequeño'] \\
\hline ulo & $\begin{array}{l}\text { ulu }(m) \text { lat., hace adj. o sust., } \\
\text { 'pequeño'. }\end{array}$ & $\begin{array}{l}\text { glomérulo, túbulo, } \\
\text { acetábulo, nódulo, lóbulo. }\end{array}$ & $\begin{array}{l}\text { [glomer- lat. 'apelotonamiento', 'bola' + } \\
u l u(m) \text { lat. 'pequeño'] }\end{array}$ \\
\hline \multicolumn{4}{|c|}{ Abundancia } \\
\hline os & $\begin{array}{l}\overline{o s}(u m) / \overline{o s}(\mathrm{am}) \text { lat., } \\
\text { adj., 'relacionado } \\
\text { 'abundancia' }\end{array}$ & 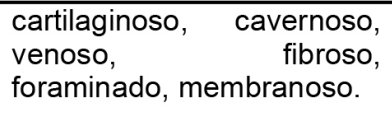 & 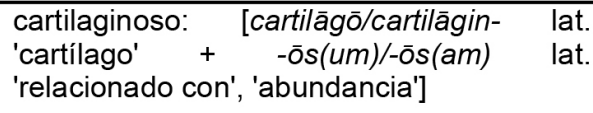 \\
\hline \multicolumn{4}{|c|}{ Que hace algo } \\
\hline nte & $\begin{array}{l}\text { nte }(m) \text { lat., hace participios, } \\
\text { 'que hace' }\end{array}$ & $\begin{array}{l}\text { aferente, } \\
\text { deferente. }\end{array}$ & $\begin{array}{l}\text { aferente: [ad lat. 'junto a', 'hacia' + fer(re) } \\
\text { lat. 'llevar' + -nte }(m) \text { lat. 'que hace'] }\end{array}$ \\
\hline dor(a), tor & $\begin{array}{l}\text { tōr(em) lat., hace sust., 'que } \\
\text { hace' }\end{array}$ & $\begin{array}{l}\text { aductor, obturador, } \\
\text { abductor, elevador. }\end{array}$ & $\begin{array}{l}\text { aductor: [ad lat. 'junto a', 'hacia' + } d \bar{u} c(e r e) \\
\text { lat. 'conducir', 'llevar' + -tōr(em) lat. 'que } \\
\text { hace'] }\end{array}$ \\
\hline
\end{tabular}

Los sufijos apreciativos del tipo -hacen algo-, están presentes en $T A$ con las terminaciones nte, dor $(a)$, tor. Estos permiten la formación de términos como, por ejemplo: eferente, deferente, aductor, obturador, abductor, corrugador, elevador, entre otros. Estos sufijos permiten otorgar la característica de acción "llevar" o "conducir". Un ejemplo de esto pude ser el músculo aductor largo, donde el término aductor indica que el músculo cumple una acción de "llevar" o "conducir" hacia la aducción.

En cuanto al idioma oficial de TA, la FIPAT es clara en transmitir que este debe ser el latín. Sin embargo, no deja de llamar la atención que los términos allí incluidos poseen sus orígenes en la antigua Grecia y Roma (Sakai), con una fuerte 
influencia del latín clásico y el griego (Wulff, 2004). Es así como nuestros resultados reafirman este postulado, al demostrar que los prefijos y sufijos utilizados para la descripción anatómica, tienen un origen compartido entre latín y el griego. Lo interesante es que independiente del origen de cada afijo, estos cumplen fielmente su función en la construcción y sentido de los términos anatómicos. Un claro ejemplo de ello son los términos "subtálamo" e "hipotálamo", dos estructuras distintas que conforman parte del diencéfalo y se ubican bajo el tálamo. La única diferencia de estos dos términos en cuanto al lenguaje es la procedencia de su prefijo (Cortés \& Ureña).

Ahora bien, para aplicar estos resultados es importante definir el aprendizaje como un cambio de conducta más o menos permanente, lo cual es producto de la experiencia o las vivencias. Para que este cambio se produzca, es necesario que el sujeto transforme la información que recibe, por lo tanto, no se trata sólo de captar datos, sino más bien, de hacer con ellos un trabajo de construcción (Martínez, 1994). Por tanto, con este trabajo se busca que el estudiante logre comprender la construcción del término anatómico, pudiendo así alcanzar un aprendizaje significativo.

Finalmente, este estudio analiza y expone el uso de prefijos y sufijos en $T A$, estableciendo que cada uno de ellos logra dar claramente un sentido a las palabras de las cuales forma parte, llegándose a hipotetizar que el conocimiento sobre esta materia por parte de docentes y estudiantes podría favorecer los procesos de enseñanza y aprendizaje de la anatomía humana.

VIDAL-SEGUEL，N.; PUMEYRAU-SOLAR. M.; LIZAMA-PÉREZ, R. \& VÁSQUEZ, B. Use of prefixes and suffixes in Terminologia Anatomica. An analysis from an etymological origin. Int. J. Morphol., 39(3):698-704, 2021.

SUMMARY: Learning anatomy requires students to incorporate a significant amount of new vocabulary, which may lead to some difficulties as students are unfamiliar with most of these terms. Despite efforts realized over time to make the use of Terminologia Anatomica (TA) clear and precise, teaching anatomy remains a complex issue. In this context, it becomes necessary to develop innovative new strategies that favor student learning. The objective of this study therefore, was to analyze, from an etymological origin, the prefixes and suffixes included in the $T A$, in order to facilitate the teaching and learning processes of human anatomy. The use of each prefix and suffix in anatomy was investigated in the second edition of the TA. The affixes found were analyzed from an etymological point of view according to the guidelines proposed by the Medical-Biological, Historical and Etymological Dictionary of the University of Salamanca. As a result, it was possible to corroborate a wide use of prefixes and suffixes in $T A$, and verify that all the terms analyzed from an etymological point of view, coincide with their current use in anatomy. Consequently, their knowledge and understanding could facilitate the teaching and learning processes of human anatomy.

KEY WORDS: Prefixes; Suffixes; Terminologia Anatomica; Education; Human anatomy.

\section{REFERENCIAS BIBLIOGRÁFICAS}

Aziz, M. A.; McKenzie, J. C.; Wilson, J. S.; Cowie, R. J.; Ayeni, S. A. $\&$ Dunn, B. K. The human cadaver in the age of biomedical informatics. Anat. Rec., 269(1):20-32, 2002.

Bergman, E. M.; de Bruin, A.; Herrler, A.; Verheijen, I. W.; Scherpbier, A. J. \& van der Vleuten, C. P. Students' perceptions of anatomy across the undergraduate problem-based learning medical curriculum: a phenomenographical study. BMC Med. Educ., 13:152, 2013.

Cortés, F. \& Ureña, J. Diccionario médico-biológico, histórico y etimológico. Salamanca, Ediciones Universidad de Salamanca, 2019.

Federative International Programme for Anatomical Terminology (FIPAT). Terminologia Anatomica. $2^{\text {nd }}$ ed. FIPAT.library.dal.ca. Federative International Programme for Anatomical Terminology, 2019.

Guiraldes, H.; Oddó, H.; Mena, B.; Velasco, N. \& Paulos, J. Enseñanza de la anatomía humana: experiencias y desafíos en una escuela de medicina. Rev. Chi. Anat., 19(2):205-12, 2001.

Jiménez, A. M. E. Afijos grecolatinos y de otra procedencia en términos médicos. MEDISAN, 16(6):1005, 2012.

Kroeger, P. R. Analyzing Grammar an Introduction. United states of America, Cambridge University Press, 2005.

Kulkarni, J. Importance of cadaver dissection-A brief review report. S.M.U. Med. J., 1:128-31, 2014.

Lizama-Pérez, R.; Vidal-Seguel, N.; Pumeyrau-Solar, M.; MuñozCofré, R.; Conei, D.; Nicholson, C. \& Vásquez, B. ¿Lobo o Lóbulo? Una explicación desde el origen etimológico: Propuesta para Terminologia Anatomica. Int. J. Morphol., 38(4): 1047-52, 2020.

Marcos, M. F. Curso de gramática española. España, cincel. S.A, 1980.

Martínez, S. G. C. La memoria y su relación con el aprendizaje. Sinéctica, 4:1-11, 1994.

Moya, M. P.; Contreras, M. \& del Sol, M. Lexema phy-sis $\phi v \sigma ı \varsigma$ en Terminologia Anatomica. Int. J. Morphol., 35(3):919-24, 2017.

Moore, K. L.; Dalley, A. F.; Agur, A. M. R. Anatomía con orientación Clínica. $6^{\mathrm{a}}$ ed. España, Lippincott Williams \& Wilkins, 2010.

Real Academia Nacional de Medicina. Diccionario de términos médicos. Buenos Aires, Ed. Médica Panamericana, 2011.

Sakai, T. Historical evolution of anatomical terminology from ancient to modern. Anat. Sci. Int., 82(2):65-81, 2007.

Singh, R.; Tubbs, R. S.; Gupta, K.; Singh, M.; Jones, D. G. \& Kumar, $\mathrm{R}$. Is the decline of human anatomy hazardous to medical education/profession?- A review. Surg. Radiol. Anat., 37(10):125765, 2015.

Smith, S. B.; Carmichael, S. W.; Pawlina, W. \& Spinner, R. J. Latin and Greek in gross anatomy. Clin. Anat., 20(3):332-7, 2007.

Stephens, S. \& Moxham, B. The attitudes of medical students toward the importance of understanding classical Greek and Latin in the development of an anatomical and medical vocabulary. Clin. Anat., 29(6):696-701, 2016. 
VIDAL-SEGUEL, N.; PUMEYRAU-SOLAR. M.; LIZAMA-PÉREZ, R. \& VÁSQUEZ, B. Uso de prefijos y sufijos en Terminologia Anatomica. Un análisis desde el origen etimológico. Int. J. Morphol., 39(3):698-704, 2021

Torres, N. Y. M. Enseñanza de anatomía: Una experiencia a partir de cuestionamientos propuestos en situaciones contextuales. Escenarios, 11(1):131-8, 2013.

Vidal-Seguel, N.; Pumeyrau-Solar, M.; Lizama-Pérez, R.; Muñoz-Cofré, R.; Conei, D.; Nicholson, C. \& del Sol, M. Análisis del Lexema Neur(o) y sus Derivados en el Aprendizaje de la Anatomía. Int. J. Morphol., 37(4):1517-21, 2019.

Vidal-Seguel, N.; Miranda, K. R. E.; Pumeyrau-Solar, M. \& Vásquez, B. Definiciones de los reparos, marcas óseas o accidentes de la superficie del hueso en Anatomía Humana. Int. J. Morphol., 39(1):335-40, 2020.

Wulff, H. R. The language of medicine. J. R. Soc. Med., 97(4):187-8, 2004.
Dirección para correspondencia:

Dra. Bélgica Vásquez

Universidad de Tarapacá

Iquique

CHILE

E-mail: bvasquezp@uta.cl

Dirección para correspondencia:

Nicolás Enrique Vidal Seguel

Universidad Santo Tomás

Los Ángeles

CHILE

E-mail: nvidal4@santotomas.cl

Recibido : 13-10-2020

Aceptado: 09-03-2021 